\title{
Self-Diffusion in Amorphous Silicon
}

\author{
Florian Strauß, ${ }^{1, *}$ Lars Dörrer, ${ }^{1}$ Thomas Geue, ${ }^{2}$ Jochen Stahn, ${ }^{2}$ Alexandros Koutsioubas, ${ }^{3}$ \\ Stefan Mattauch, ${ }^{3}$ and Harald Schmidt ${ }^{1}$ \\ ${ }^{1}$ AG Mikrokinetik, Institut für Metallurgie, TU Clausthal, D-38678 Clausthal-Zellerfeld, Germany \\ ${ }^{2}$ Laboratory for Neutron Scattering and Imaging, Paul Scherrer Institute, Villigen CH-5232, Switzerland \\ ${ }^{3}$ Jülich Centre for Neutron Science (JCNS), Forschungszentrum Jülich GmbH, Outstation at MLZ, \\ Lichtenbergstraße 1, D-85747 Garching, Germany \\ (Received 12 August 2015; revised manuscript received 30 October 2015; published 13 January 2016) \\ The present Letter reports on self-diffusion in amorphous silicon. Experiments were done on ${ }^{29} \mathrm{Si} /{ }^{\text {nat }} \mathrm{Si}$ \\ heterostructures using neutron reflectometry and secondary ion mass spectrometry. The diffusivities follow \\ the Arrhenius law in the temperature range between 550 and $700{ }^{\circ} \mathrm{C}$ with an activation energy of \\ $(4.4 \pm 0.3) \mathrm{eV}$. In comparison with single crystalline silicon the diffusivities are tremendously higher by 5 \\ orders of magnitude at about $700^{\circ} \mathrm{C}$, which can be interpreted as the consequence of a high diffusion \\ entropy.
}

DOI: 10.1103/PhysRevLett.116.025901

The technological importance of amorphous silicon ( $a$-Si) easily leads to the conclusion that the material is well understood. Yet, although it is widely used in everyday items such as solar cells [1], thin film transistors [2], thin film display technologies [3], light emission diodes [4] and even lithium-ion batteries [5], reliable experimental data on self-diffusion do not exist. For amorphous solids such data are of high importance because atomic mobility determines the intrinsic thermal stability of these materials and, consequently, also their range of use in technical applications. In literature, there exists some theoretical work on the simulation of a-Si self-diffusion by molecular dynamics $[6,7]$. Some publications are also available that investigate interdiffusion in $a-\mathrm{Si} / a-\mathrm{Ge}$ multilayer sample [8]. These results can be used to deduce upper limits of the selfdiffusivity in a-Si but have to be handled with care due to the presence of chemical gradients, density changes, and mechanical stress. On the other hand, self-diffusion in crystalline silicon has been experimentally investigated for decades $[9,10]$. In a recent publication on this topic even the temperature range below $700^{\circ} \mathrm{C}$ is probed where corresponding amorphous material would not show an onset of crystallization [11]. A direct comparison of selfdiffusivities in the crystalline and amorphous state will give insight into differences and similarities concerning kinetics and defect structure and is of fundamental and broad interest.

As a model system for a covalently bound amorphous semiconductor, a-Si can best be described as a fourfold coordinated continuous random network of silicon atoms [12], exhibiting a deviation in bond lengths and bond angles from the values in its crystalline form [13]. The periodicity of the crystalline structure is not present in the amorphous state, but the short range order is comparable. Because of the statistical nature of the network there are about $20 \%$ fivefold coordinated $\mathrm{Si}$ atoms, resulting in dangling bonds on other sites [7]. These dangling bonds are possible recombination centers for charge carriers [14] and often passivated by hydrogen, forming a-Si:H [15].

a-Si can be produced, e.g., by laser amorphization of crystalline silicon [16], chemical vapor deposition [17], ion-beam irradiation [18], magnetron sputtering [19], or ion-beam sputtering [20]. Independent of the production method, a-Si is thermodynamically in a nonequilibrium state. Thus, self-diffusion is an important factor in thermally activated structural reorganization processes such as crystallisation or relaxation towards intermediate metastable equilibria. At temperatures well below the onset of crystallization, a-Si undergoes a reorganization process referred to as structural relaxation [21,22]. This means an increase in short range order with an accompanying reduction in free energy but still maintaining the amorphous state [21]. Since atomic mobility is assumed to govern these phenomena, a better understanding of diffusion, primarily by knowing the corresponding diffusivities, is the first step to a better description of this material. In addition, self-diffusion phenomena in a-Si also exhibit a strong influence on mechanical stability and plastic deformation, which is of special interest considering electrodes in Li-ion batteries, where the volume expansion of up to $400 \%$ during lithiation is a major problem [5].

The lack of reliable diffusivities in a-Si can mainly be ascribed to the previously mentioned metastability. This necessitates experiments at low temperatures, probing for small diffusion lengths in the nanometer range and low diffusivities well below $10^{-20} \mathrm{~m}^{2} / \mathrm{s}$. It has already been shown that neutron reflectometry (NR) offers a toolset for such problems [23]. Consequently, the aim of the present Letter is the determination of self-diffusivities of a-Si with NR. In order to achieve this goal, characteristic samples have to be produced suitable for neutron experiments. The interaction of neutrons with the atomic nucleus gives rise to 
a sensitivity to stable isotopes of a certain element in contrast to the sensitivity of $\mathrm{x}$ rays for electrons and, consequently, for chemical differences only. Thus isotope multilayers are produced for the neutron experiments, meaning the alternating deposition of ${ }^{\text {nat }} \mathrm{Si}$ (mainly composed of ${ }^{28} \mathrm{Si}$ ) and an isotopically enriched $\mathrm{Si}$ species. Here, ${ }^{29} \mathrm{Si}$ was used, which has a neutron scattering length of $4.70 \mathrm{fm}$ that differs sufficiently from the $4.15 \mathrm{fm}$ of ${ }^{\text {nat }} \mathrm{Si}$ to realize suitable multilayer samples. The isotope periodicity of those multilayers gives rise to artificial Bragg peaks in the reflectivity pattern of neutrons. The decrease of such peaks, e.g., after annealing steps, can then be used to determine diffusivities down to $10^{-25} \mathrm{~m}^{2} / \mathrm{s}[23,24]$.

For the presented experiments, amorphous $\left[{ }^{29} \mathrm{Si}(7 \mathrm{~nm}) \mid{ }^{\text {nat }} \mathrm{Si}(24 \mathrm{~nm})\right] \times 10$ multilayer structures were produced by ion-beam sputter deposition onto commercial (100) oriented silicon wafers. For additional experiments with secondary ion mass spectrometry (SIMS) as an alternative analysis method to probe diffusion at higher temperatures, amorphous bilayer samples of $50 \mathrm{~nm}^{29} \mathrm{Si}$ on top of $120 \mathrm{~nm}{ }^{\text {nat }} \mathrm{Si}$ were used. Detailed information on experimental procedures can be found in the Supplemental Material [25].

Structural investigation was done by grazing-incidence x-ray diffractometry (GI-XRD) measurements of sputtered silicon films in the as-deposited state and at $700{ }^{\circ} \mathrm{C}$, which is the highest temperature under investigation (see Fig. S1 in the Supplemental Material [25]). The as-deposited sample is clearly x-ray amorphous. Annealing for $3 \mathrm{~min}$ at $700{ }^{\circ} \mathrm{C}$ does not change the pattern. In contrast, the curve for the sample annealed for $1 \mathrm{~h}$ at $700^{\circ} \mathrm{C}$ shows three distinct Bragg peaks which can be attributed to 111, 220, and 311 reflections. Further synchrotron based in situ measurements using a monochromatic beam also showed no sign of crystallinity [20]. Additionally, high resolution transmission electron microscopy (HR-TEM) measurements of an as-deposited bilayer structure as used for the SIMS experiments confirm the amorphous state. For a sample annealed for $1 \mathrm{~h}$ at $650^{\circ} \mathrm{C}$, HR-TEM shows some isolated nanocrystallites with a diameter of $\sim 5 \mathrm{~nm}$ which do not form a percolation path within the amorphous matrix. These nanocrystals are not visible in the GI-XRD data. The combination of these results confirms that diffusion is measured in an amorphous structure.

In Fig. 1(a) a typical reflectivity pattern of an as-deposited sample as obtained by NR is shown. The pattern exhibits the edge of total reflection at $0.011 \AA^{-1}$ and a Bragg peak caused by the isotope modulation at $0.0234 \AA^{-1}$. The pattern is a superposition of the Fresnel reflectivity of silicon with the interference pattern characteristic of the multilayer structure. For background correction, the reflectivity of the amorphous sample, $R_{\text {sample }}$, is divided by the measured reflectivity of a conventional silicon wafer, $R_{\text {wafer }}$. This is shown in Fig. 1(b) for an as-deposited sample and a sample from the same batch treated for $1040 \mathrm{~min}$ at $600^{\circ} \mathrm{C}$. A more
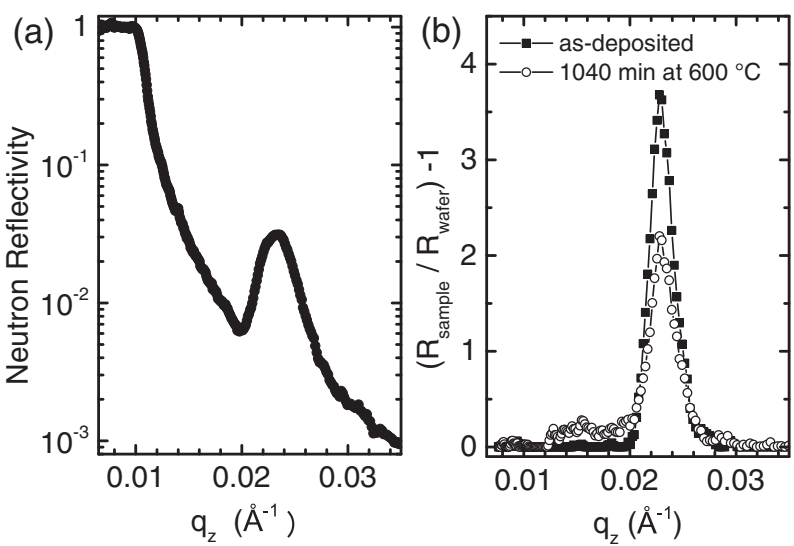

FIG. 1. (a) Typical neutron reflectivity pattern of an amorphous ${ }^{29} \mathrm{Si} /{ }^{\text {nat }} \mathrm{Si}$ multilayer sample (logarithmic scale). A moving average of four data points each was used on the raw data. (b) Comparison of neutron reflectivities of an as-deposited sample (black squares) and a sample annealed for $1040 \mathrm{~min}$ at $600{ }^{\circ} \mathrm{C}$ plotted against the scattering vector $q_{z}$. Reflectivities are corrected by dividing by the Fresnel reflectivity of a Si wafer and subtracting one.

detailed description of NR data evaluation is given in the Supplemental Material [25]. A decrease in Bragg peak intensity (integrated area below the peak), $I(t)$, is clearly visible, which can be used to calculate self-diffusivities according to Refs. $[23,26]$ using the following equation:

$$
I(t)=I_{S}+\left(I_{0}-I_{S}\right) \exp \left(-\frac{8 \pi^{2} t}{l^{2}} D\right)
$$

Here, $I_{0}$ is the intensity of the Bragg peak at time $t=0$ (as-deposited sample) and $l=31 \mathrm{~nm}$ is the bilayer periodicity. $I_{S}=0.4$ stands for a saturation intensity [20] which will be explained in the discussion below. A comparison of peak intensities in Fig. 1(b) gives a decrease to about $49 \%$ of the initial intensity for the annealed sample. This is equivalent to a self-diffusivity of $(3.9 \pm 2.2) \times 10^{-22} \mathrm{~m}^{2} / \mathrm{s}$. The diffusivities obtained at different temperatures are plotted in Fig. 2 as a function of inverse temperature and are listed in Table SI of the Supplemental Material [25]. An annealing time dependence of diffusivities was not found. Thus, the concentration of point defects governing diffusion is not modified within error limits (e.g., by structural relaxation) during annealing at constant temperature. According to Stolk et al. [27], structural relaxation processes have been shown to be completed in a timeframe of several seconds at temperatures used in the given experiments. Consequently, an experimental observation is beyond the scope of our experimental equipment at the moment. Structural relaxation is completed before its effect on diffusion can be detected. In which way structural relaxation may influence diffusion needs to be the subject of future studies at lower temperatures, where the timeframe of observation is larger. 


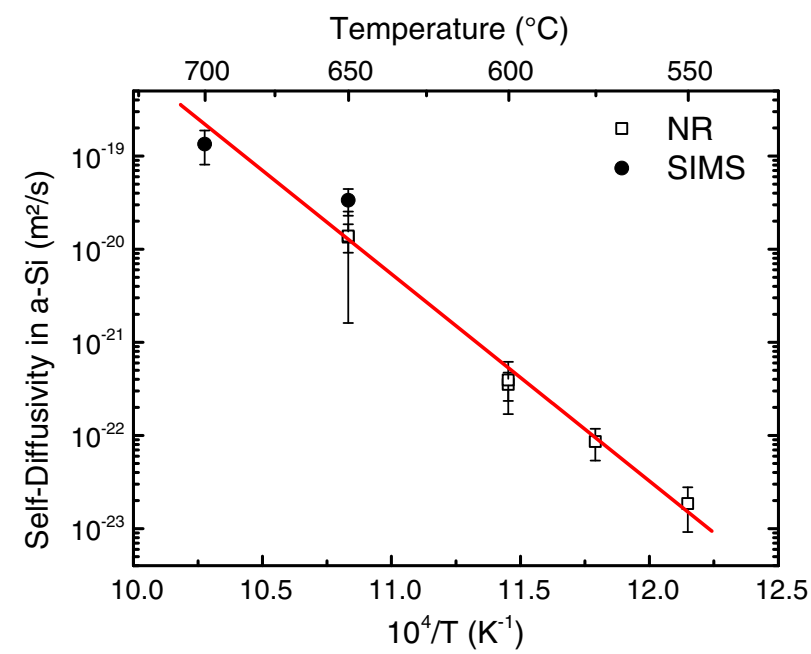

FIG. 2. Self-diffusivities in amorphous silicon plotted against the reciprocal temperature. The straight line depicts a linear fit to the data according to Eq. (2).

In addition to NR, supplementary experiments were done by SIMS at temperatures of 650 and $700^{\circ} \mathrm{C}$ on isotope bilayers. Figure 3 shows a comparison of the relative ${ }^{29} \mathrm{Si}$ isotope fraction, $c$, for the as-deposited sample and a sample from the same batch, annealed at $700^{\circ} \mathrm{C}$ for 3 min. Diffusion is reflected in a broadening of the whole profile of the annealed sample, which is clearly visible. Diffusivities can be determined as described in the Supplemental Material [25]. The slight deviation of the fit from the data points in the lower bend of the curve is attributable to ion-beam mixing. An evaluation of the data of Fig. 3 yields a self-diffusivity of $(1.3 \pm 0.5) \times 10^{-19} \mathrm{~m}^{2} / \mathrm{s}$. The SIMS results confirm the NR results at $650^{\circ} \mathrm{C}$ within error limits, proving the reliability of the neutron based data.

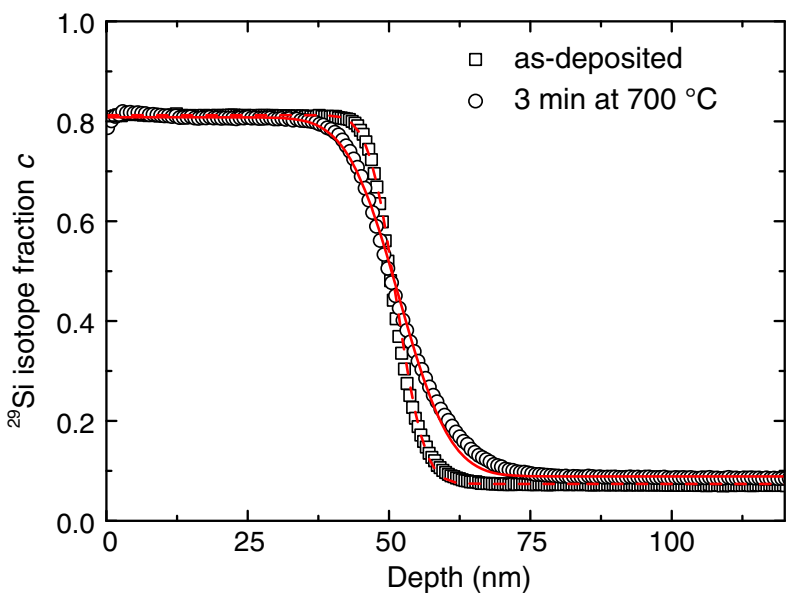

FIG. 3. Relative ${ }^{29} \mathrm{Si}$ fraction as a function of sputter depth of ${ }^{29} \mathrm{Si} /{ }^{\text {nat }} \mathrm{Si}$ bilayer. The squares represent the as-deposited sample while the circles show the data of a sample annealed for $3 \mathrm{~min}$ at $700{ }^{\circ} \mathrm{C}$. The lines represent the fits according to Eq. (S1) of the Supplemental Material [25] for the as-deposited and annealed case, respectively.
The saturation intensity $I_{S}$ introduced above for the analysis of the NR measurements is attributable to the carbon contamination that is present in the ${ }^{29} \mathrm{Si}$ layer. This carbon results in an additional rise of the scattering length density of the ${ }^{29} \mathrm{Si}$ layer and to a further contribution to the Bragg peaks. As shown in Ref. [20], carbon stays localized in the ${ }^{29} \mathrm{Si}$ layer, even for the longest annealing times presented here. Consequently, its contribution to the Bragg peak does not change during annealing and the Bragg peak does not vanish completely during annealing. Furthermore, carbon is an isoelectronic element to silicon most likely forming immobile $\mathrm{SiC}$ clusters in the ${ }^{29} \mathrm{Si}$ layers. A look at available literature shows a virtual immobility of silicon in $\mathrm{SiC}$ in the temperature range investigated [28]. Support can also be found in the SIMS measurements on the bilayer structures as given in Fig. 3. No measurable asymmetry in the diffusion profile after annealing can be found, which would be visible if a significant influence of the immobile carbon on the Si diffusion is present. It is to be noted that we do not exclude a priori an influence of carbon on $\mathrm{Si}$ diffusion, but for the present experiment it is below the detection limit.

The diffusivities given in Fig. 2 follow the Arrhenius law given as

$$
D=D_{0} \exp \left(-\frac{E_{a}}{k_{B} T}\right)
$$

The applied straight line fit yields an activation energy of $(4.4 \pm 0.3) \mathrm{eV}$ and a preexponential factor of $D_{0}=1.5 \times$ $10^{5} \mathrm{~m}^{2} / \mathrm{s}$ (error: $\log _{10} D_{0}=1.5$ ).

This comparably high value of $4.4 \mathrm{eV}$ indicates that the activation energy of diffusion has to be composed of a migration and a defect formation part. In literature, data on activation energies of diffusion are very limited. A theoretical study on self-diffusion in amorphous silicon can be found which was done between 627 and $1027^{\circ} \mathrm{C}$ by classical molecular dynamics [7]. An activation energy of silicon migration between 0.86 and $0.95 \mathrm{eV}$ is derived. In a different study [29], a migration energy of only $0.23 \mathrm{eV}$ is calculated at low temperatures between 27 and $327^{\circ} \mathrm{C}$. Mirabella et al. [30] give an activation energy of dangling bond migration (as the underlying diffusion defect) of $2.6 \mathrm{eV}$ as derived from boron diffusion in a-Si. Further information on activation energies in a-Si can be found in experiments investigating structural relaxation, e.g., by resistivity measurements. This process involves point defect annihilation during annealing, necessitating a movement of atoms. According to various literature work [31], activation energy spectra are found, ranging between 0.2 and $2.7 \mathrm{eV}$, which should at most represent the migration part of our activation energy given above. The spectral aspect of the activation energies for structural relaxation hints at a complex diffusion process with varying local coordination and orientation. 
In conclusion, literature data give estimations of migration energies of defects up to a maximum value of $2.7 \mathrm{eV}$. This confirms our assumption above that our activation energy of diffusion of $4.4 \mathrm{eV}$ is the sum of a defect formation and migration part. This finding shows that structural, temperature-independent defects do not play an important role in the temperature range investigated. Otherwise, a lower activation energy, solely composed of the migration part would be obtained. With all necessary care, we can assess from the available data a defect formation energy of at least $1.7 \mathrm{eV}$ (lower limit).

For further analysis, the present results are compared to the diffusion parameters of single crystalline silicon as found in the literature [11]. The diffusivities in a-Si are 5 orders of magnitude higher than in single crystals if compared at $700{ }^{\circ} \mathrm{C}$ (the approximate crystallization temperature). This is a tremendous difference which is not found for other amorphous semiconductors such as silicon nitride [32] and has to be discussed further. First, the activation energies are compared. The activation energy of self-diffusion in crystalline silicon is given as $4.95 \mathrm{eV}$ for the contribution of interstitials [11] and as $3.6 \mathrm{eV}$ for the contribution of vacancies [10]. Alternatively, a temperature dependent activation energy of vacancies is postulated in Ref. [11], which is less than $3.6 \mathrm{eV}$ below $700^{\circ} \mathrm{C}$. At temperatures above $900^{\circ} \mathrm{C}$ the interstitial mechanism is prevalent, while for lower temperatures a significant influence of vacancies is found. In general, diffusion in amorphous materials is expected to yield a lower activation energy compared to its crystalline counterpart, chiefly attributable to a more open structure and easier defect formation. For the present case, when comparing activation energies, this points to diffusion in amorphous silicon taking place by an interstitial-like mechanism (in the broadest sense). Under this assumption, the activation energies of 4.95 (crystalline) and $4.4 \mathrm{eV}$ (amorphous) are quite similar.

The main reason for the tremendous difference in diffusivities becomes evident by contrasting the preexponential factors of $1.5 \times 10^{5} \mathrm{~m}^{2} / \mathrm{s}$ for amorphous silicon and $2.2 \mathrm{~m}^{2} / \mathrm{s}$ for crystalline silicon. The extremely high preexponential factor of a-Si is difficult to understand. Yet, from research on metallic glasses [33] it is known that $D_{0}$ can differ by several orders of magnitude between the crystalline and amorphous phase of the same type of material, including high preexponential factors. This is mainly attributed to a high entropy of diffusion, often resulting from the participation of several atoms in a single jump [33]. From our preexponential factor an entropy of diffusion as high as $\Delta S \approx 26 \mathrm{k}_{B}$ can be assessed, using $\Delta S \approx \ln \left(D_{0} / a^{2} \nu\right)$ and $a=2.35 \AA$ as the $\mathrm{Si}-\mathrm{Si}$ atomic distance and $\nu \approx 1.3 \times 10^{13} \mathrm{~s}^{-1}$ as the Debye frequency [34]. An explanation of the relatively high activation entropy of self-diffusion in crystalline $\mathrm{Si}$ and $\mathrm{Ge}$ (compared to metals) as spread-out point defects was first proposed by
Seeger and Chik $[11,35]$. More insight gives recent work on extended point defects in crystalline Ge and Si by Cowern et al. [36]. These authors postulate the presence of two distinct self-interstitial forms in order to explain $B$ diffusion in crystalline Ge: A simple interstitial with a low entropy of diffusion to be present at low temperatures and a complex one with an entropy of $30 \mathrm{k}_{B}$ to be present at high temperatures. This concept proved useful in interpreting our results, but is also critically discussed by a different group [37] (for details of this open discussion cf. Ref. [38]). The structure of this extended defect [36] is assumed to be similar to an amorphous pocket, a complex thermodynamically stable structure incorporating several atoms of the lattice. The self-interstitial is extended over a certain volume of the lattice ( $\mathrm{N}$ atoms occupy a volume normally occupied by $\mathrm{N}-1$ lattice atoms). The authors further predict by a semiempiric model an activation energy of diffusion of about $6 \mathrm{eV}$ for interstitial based extended defects and of about $5 \mathrm{eV}$ for vacancy based extended defects in crystalline silicon. The corresponding entropy of diffusion is given as $16 \mathrm{k}_{B}$ and $9 \mathrm{k}_{B}$, respectively. These defects move by shape shifting through numerous configurations [36]. In light of these results, we interpret our data that such an extended defect structure might also be governing diffusion in $a$-Si. However, since the formation of this vehicle does not start from a crystalline structure but from the amorphous structure (necessarily different from the extended defect structure) the corresponding formation energies and consequently activation energies are expected to be lower. This would be in accordance with the activation energy of self-diffusion of $4.4 \mathrm{eV}$ found in this study.

In literature, the main defects in $a$-Si that are thought to contribute to diffusion are dangling bonds [39] and floating bonds [40]. Moreover, other atomic rearrangement processes via bond break, bond switch, or even interstitial-like configurations or vacancylike defects $[7,41]$ are suggested in theoretical work. As of yet, there is no consensus and no experimental evidence on which of these defects play a role in self-diffusion processes in $a-\mathrm{Si}$ [40]. However, a preferred idea is the dangling bond as an active site for diffusing species [42]. In Ref. [43] the formation energy of a dangling bond in $a-\mathrm{Si}$ is assessed from experiments to be around $1 \mathrm{eV}$ by applying the concept of thermalization energy to bias-stress data of thin film transistors. This value would be too low to explain our results (see above). In light of the extended defect picture sketched above and given in (Ref. [36], Supplemental Material [25]), such a defect diffuses by successive rebonding events at its interface, resulting in a shift of its center of mass. Here, also dangling bonds might be involved.

In conclusion, we reported on measurements of selfdiffusion in amorphous silicon using neutron reflectometry on ${ }^{29} \mathrm{Si} /{ }^{\text {nat }} \mathrm{Si}$ isotope multilayers. Diffusivities between $10^{-23}$ and $10^{-19} \mathrm{~m}^{2} / \mathrm{s}$ for temperatures between 550 and $700{ }^{\circ} \mathrm{C}$ were measured, following the Arrhenius law. The 
diffusion mechanism in itself is not completely understood, but the comparably high activation energy of (4.4 \pm $0.3) \mathrm{eV}$ indicates the contribution of a migration and a defect formation part. A comparison to crystalline silicon yields significantly higher diffusivities for $a$-Si at a given temperature due to a high diffusion entropy but a comparable activation energy. As a possible reason for the high diffusion entropy, the presence of extended point defects incorporating several atoms of the lattice is suggested.

This work is based upon experiments performed at the Swiss spallation neutron source SINQ, Paul Scherrer Institute, Villigen, Switzerland and at the neutron reflectometer MARIA, operated by JCNS at Heinz MaierLeibnitz Zentrum, Garching, Germany. This research project has been supported by the German Research Foundation under the contract Schm1569/22-1 and by the European Commission under the 7th Framework Programme through the "Research Infrastructures" action of the "Capacities" Programme, NMI3-II Grant No. 283883. The TEM measurements mentioned in this work were carried out by C. Kübel with the support of the Karlsruhe Nano Micro Facility (KNMF, www.knmf.kit .edu). The authors thank G. Borchardt for the use of his SIMS equipment.

*florian.strauss@tu-clausthal.de

[1] M. Kondo, T. Matsui, Y. Nasuno, H. Sonobe, and S. Shimizu, Thin Solid Films 501, 243 (2006); A. Shah, P. Torres, R. Tscharner, N. Wyrsch, and H. Keppner, Science 285, 692 (1999).

[2] H. Gleskova and S. Wagner, IEEE Electron Device Lett. 20, 473 (1999); M. J. Powell, IEEE Trans. Electron Devices 36, 2753 (1989).

[3] A. Nathan, A. Kumar, K. Sakariya, P. Servati, S. Sambandan, and D. Striakhilev, IEEE J. Solid-State Circuits 39, 1477 (2004).

[4] P. Servati, S. Prakash, A. Nathan, and C. Py, J. Vac. Sci. Technol. A 20, 1374 (2002).

[5] M. T. McDowell, S. W. Lee, W. D. Nix, and Y. Cui, Adv. Mater. (Weinheim, Ger.) 25, 4966 (2013).

[6] G. T. Barkema, N. Mousseau, R. Vink, and P. Biswas, MRS Proc. 664, A28.1 (2001).

[7] I. Santos, L. A. Marqués, L. Pelaz, and L. Colombo, Phys. Rev. B 83, 153201 (2011).

[8] C. Janot, A. Bruson, and G. Marchal, J. Phys. (Les Ulis, Fr.) 47, 1751 (1986); S. M. Prokes and F. Spaepen, Appl. Phys. Lett. 47, 234 (1985); A. Csik, G. A. Langer, D. L. Beke, Z. Erdélyi, M. Menyhard, and A. Sulyok, J. Appl. Phys. 89, 804 (2001); M. A. Noah, D. Flötotto, Z. Wang, and E. J. Mittemeijer, J. Appl. Phys. 117, 165306 (2015).

[9] H. Bracht, E. E. Haller, and R. Clark-Phelps, Phys. Rev. Lett. 81, 393 (1998); J. M. Fairfield and B. J. Masters, J. Appl. Phys. 38, 3148 (1967); L. Kalinowski and R. Seguin, Appl. Phys. Lett. 35, 211 (1979); A. Ural, P. B. Griffin, and J. D. Plummer, Phys. Rev. Lett. 83, 3454 (1999).
[10] Y. Shimizu, M. Uematsu, and K. M. Itoh, Phys. Rev. Lett. 98, 095901 (2007).

[11] R. Kube, H. Bracht, E. Hüger, H. Schmidt, J. L. Hansen, A. N. Larsen, J. W. Ager, E. E. Haller, T. Geue, and J. Stahn, Phys. Rev. B 88, 085206 (2013).

[12] D. E. Polk and D. S. Boudreaux, Phys. Rev. Lett. 31, 92 (1973).

[13] S. R. Elliott, Adv. Phys. 38, 1 (1989); S. Kugler and Z. Várallyay, Philos. Mag. Lett. 81, 569 (2001).

[14] C. Boehme, F. Friedrich, T. Ehara, and K. Lips, Thin Solid Films 487, 132 (2005).

[15] R. A. Street, Physica (Amsterdam) 170B, 69 (1991); W. Beyer and U. Zastrow, J. Non-Cryst. Solids 227-230, 880 (1998).

[16] T. Sameshima and S. Usui, J. Appl. Phys. 70, 1281 (1991).

[17] R. E. I. Schropp, K. F. Feenstra, E. C. Molenbroek, H. Meiling, and J. K. Rath, Philos. Mag. B 76, 309 (1997).

[18] R. G. Elliman, J. S. Williams, W. L. Brown, A. Leiberich, D. M. Maher, and R. V. Knoell, Nucl. Instrum. Methods Phys. Res., Sect. B 19-20, 435 (1987).

[19] F. Demichelis, A. Tagliaferro, E. Tresso, and P. Rava, J. Appl. Phys. 57, 5424 (1985).

[20] F. Strauß, T. Geue, J. Stahn, and H. Schmidt, Defect Diffus. Forum 363, 225 (2015).

[21] S. Roorda, S. Doorn, W. C. Sinke, P. M. L. O. Scholte, and E. van Loenen, Phys. Rev. Lett. 62, 1880 (1989).

[22] I. Štich, R. Car, and M. Parrinello, Phys. Rev. B 44, 11092 (1991).

[23] H. Schmidt, M. Gupta, T. Gutberlet, J. Stahn, and M. Bruns, Acta Mater. 56, 464 (2008).

[24] E. Hüger, U. Tietze, D. Lott, H. Bracht, D. Bougeard, E. E. Haller, and H. Schmidt, Appl. Phys. Lett. 93, 162104 (2008).

[25] See Supplemental Material at http://link.aps.org/ supplemental/10.1103/PhysRevLett.116.025901 for a more detailed description of experimental setups used, additional graphs and data tables, and a description of NR and SIMS data evaluation procedures.

[26] T. Mizoguchi and M. Murata, Jpn. J. Appl. Phys. 30, 1818 (1991).

[27] P. A. Stolk, F. W. Saris, A. J. M. Berntsen, W. F. van der Weg, L. T. Sealy, R. C. Barklie, G. Krötz, and G. Müller, J. Appl. Phys. 75, 7266 (1994).

[28] W. Gruber, U. Geckle, M. Bruns, and H. Schmidt, Thin Solid Films 518, 396 (2009).

[29] L. Brambilla, L. Colombo, V. Rosato, and F. Cleri, Appl. Phys. Lett. 77, 2337 (2000).

[30] S. Mirabella, D. De Salvador, E. Bruno, E. Napolitani, E. F. Pecora, S. Boninelli, and F. Priolo, Phys. Rev. Lett. 100, 155901 (2008).

[31] S. Coffa, F. Priolo, and A. Battaglia, Phys. Rev. Lett. 70, 3756 (1993); J. H. Shin and H. A. Atwater, Phys. Rev. B 48, 5964 (1993); S. Roorda, W. C. Sinke, J. M. Poate, D. C. Jacobson, S. Dierker, B. S. Dennis, D. J. Eaglesham, F. Spaepen, and P. Fuoss, Phys. Rev. B 44, 3702 (1991).

[32] H. Schmidt, U. Geckle, and M. Bruns, Phys. Rev. B 74, 045203 (2006).

[33] F. Faupel, W. Frank, M.-P. Macht, H. Mehrer, V. Naundorf, K. Rätzke, H. R. Schober, S. K. Sharma, and H. Teichler, Rev. Mod. Phys. 75, 237 (2003). 
[34] G. Kissinger and S. Pizzini, Silicon, Germanium, and Their Alloys. Growth, Defects, Impurities, and Nanocrystals (Taylor and Francis, Hoboken, 2014).

[35] A. Seeger and K. P. Chik, Phys. Status Solidi (b) 29, 455 (1968).

[36] N. E. B. Cowern et al., Phys. Rev. Lett. 110, 155501 (2013).

[37] A. Chroneos and H. Bracht, Appl. Phys. Rev. 1, 011301 (2014).

[38] N. E. B. Cowern, S. Simdyankin, J. P. Goss, E. Napolitani, D. De Salvador, E. Bruno, S. Mirabella, C. Ahn, and N. S. Bennett, Appl. Phys. Rev. 2, 036101 (2015); H. Bracht, T. Südkamp, M. Radek, and A. Chroneos, Appl. Phys. Rev. 2, 036102 (2015).
[39] W. G. Spitzer, G. K. Hubler, and T. A. Kennedy, Nucl. Instrum. Methods Phys. Res. 209-210, 309 (1983).

[40] S. T. Pantelides, Phys. Rev. Lett. 58, 1344 (1987).

[41] G. N. van den Hoven, Z. N. Liang, L. Niesen, and J. S. Custer, Phys. Rev. Lett. 68, 3714 (1992).

[42] J. M. Jacques, L. S. Robertson, K. S. Jones, M. E. Law, M. Rendon, and J. Bennett, Appl. Phys. Lett. 82, 3469 (2003); S. Mirabella, D. De Salvador, E. Bruno, E. Napolitani, E. F. Pecora, S. Boninelli, and F. Priolo, Phys. Rev. Lett. 100, 155901 (2008); S. Coffa, J. M. Poate, D. C. Jacobson, W. Frank, and W. Gustin, Phys. Rev. B 45, 8355 (1992).

[43] S. C. Deane, R. B. Wehrspohn, and M. J. Powell, Phys. Rev. B 58, 12625 (1998). 